\title{
WEIGHTED FACES OF POISSON HYPERPLANE TESSELLATIONS
}

\author{
ROLF SCHNEIDER, ${ }^{*}$ Albert-Ludwigs-Universität Freiburg
}

\begin{abstract}
We study lower-dimensional volume-weighted typical faces of a stationary Poisson hyperplane tessellation in $d$-dimensional Euclidean space. After showing how their distribution can be derived from that of the zero cell, we obtain sharp lower and upper bounds for the expected vertex number of the volume-weighted typical $k$-face $(k=2, \ldots, d)$. The bounds are respectively attained by parallel mosaics and by isotropic tessellations. We conclude with a remark on expected face numbers and expected intrinsic volumes of the zero cell.
\end{abstract}

Keywords: Poisson hyperplane tessellation; volume-weighted typical face; Palm distribution; extremal vertex number; parallel mosaic; associated zonoid; volume product

2000 Mathematics Subject Classification: Primary 60D05

Secondary 60G55

\section{Introduction}

A stationary Poisson hyperplane tessellation of Euclidean space $\mathbb{R}^{d}$ gives rise, in a natural way, to two different random polytopes, the zero cell and the typical cell. The zero cell is defined as the almost-surely unique cell of the tessellation that contains the origin. The distribution of the typical cell is the grain distribution, with respect to some centre function, of the stationary particle process defined by the cells of the tessellation. Intuitively and heuristically, the typical cell is obtained, up to translations, if within a 'large' bounded part of space we randomly choose a cell of the tessellation, giving equal weight to each of the cells, whereas the zero cell is obtained, up to a random translation, if the weights are proportional to the volume. If translations are disregarded, the distribution of the zero cell is the volume-weighted distribution of the typical cell. The distinction between the two types of random polytopes was pointed out in the early work on Poisson hyperplane processes (see [6]-[9]); in particular, Matheron [6], [7, p. 168] emphasized the different viewpoints of the 'number law' and the 'volume law'. The fact that the distributions of the zero cell and the typical cell are closely related via the volume, does not mean that both can be treated in a similar way. For example, Miles [8] found explicit formulae for the expectations of the $j$ th intrinsic volume, the number of $j$-faces and the total volume of the $j$-faces of the typical cell in the isotropic case, whereas for the zero cell, corresponding formulae for the expected $j$ th intrinsic volume (except for $j=0, d-1, d$ ) or the expected number of $j$-faces (except for $j=0,1, d$ ) are unknown if $d>3$.

The purpose of this note is to extend known results on zero cells to $k$-volume weighted typical $k$-faces of stationary Poisson hyperplane tessellations, for $k=1, \ldots, d-1$. One incentive for this was the recent investigation, by Baumstark and Last [2], of distributional properties of a stationary Poisson-Voronoi tessellation, as seen from a randomly chosen (typical)

Received 9 December 2008; revision received 5 April 2009.

* Postal address: Mathematisches Institut, Albert-Ludwigs-Universität Freiburg, Eckerstr. 1, D-79104 Freiburg, Germany. Email address: rolf.schneider@math.uni-freiburg.de 
point on a $k$-face of the tessellation. Baumstark and Last [1] have also obtained far-reaching generalizations of earlier results on gamma distributions related to stationary Poisson $k$-flats. Another motivation comes from the work of Weiss [13] and Favis and Weiss [5] on weighted cells of Poisson hyperplane tessellations (for weighting, see also [10]).

We denote the underlying probability space by $(\Omega, \boldsymbol{A}, \mathrm{P})$ and the mathematical expectation by E. Measures on a topological space $T$ are always measures on the Borel $\sigma$-algebra $\mathcal{B}(T)$ of the space.

Let $\widehat{X}$ be a stationary Poisson hyperplane process in $\mathbb{R}^{d}$, with intensity $\widehat{\gamma}>0$ and spherical directional distribution $\widehat{\varphi}$. (For the employed notions from the theory of random tessellations, we refer the reader to [11, Chapter 10].) We assume that $\widehat{\varphi}$, which is an even probability measure on the unit sphere $S^{d-1}$, is not concentrated on some great subsphere. In this case, the hyperplane process is called nondegenerate; almost surely, its hyperplanes are in general position and induce a random mosaic. By $X$ we denote this mosaic, that is, the stationary particle process of its cells, and by $X^{(k)}$ the process of its $k$-faces, for $k=0, \ldots, d$ (with $\left.X^{(d)}=X\right)$. Let $\gamma^{(k)}$ denote the intensity of $X^{(k)}$. With respect to a given centre function, the grain distribution $\mathbb{Q}^{(k)}$ of $X^{(k)}$ is defined, and a random polytope $Z^{(k)}$ with this distribution is called the typical $k$-face of the tessellation $X$. In Section 2 we give a precise definition of the weighted typical $k$-face $Z_{0}^{(k)}$ by means of a Palm distribution (we say 'weighted' briefly for ' $k$-volume weighted'). In particular, $Z_{0}^{(d)}=: Z_{0}$ is the zero cell of $X$. Roughly speaking, $Z_{0}^{(k)}$ is the $k$-face of $X$ containing 0 under the condition that a uniformly chosen random point on the union of the $k$-faces of $X$ coincides with 0 .

It is well known that the distribution of the zero cell $Z_{0}$ determines the distribution of the nondegenerate, stationary Poisson hyperplane process $\widehat{X}$. (For example, by [11, p. 504], the distribution of the radial function of $Z_{0}$ determines the associated zonoid of $\widehat{X}$, and this determines the distribution of $\widehat{X}$, by [11, Theorem 4.6.4].) In particular, the distribution of the weighted typical $k$-face is also determined by the distribution of the zero cell. Our first aim is to put this into evidence by an explicit formula. For this, we need some more notation.

For $k \in\{0, \ldots, d-1\}$, we denote by $\widehat{X}_{d-k}$ the intersection process of order $d-k$ of $\widehat{X}$ (which is induced by the intersections of any $d-k$ hyperplanes of $\widehat{X}$; see [11, Section 4.4]). Thus, $\widehat{X}_{d-k}$ is almost surely (a.s.) a stationary process of $k$-flats. We denote its intensity by $\widehat{\gamma}_{d-k}$ and its directional distribution by $\widehat{\mathbb{Q}}_{d-k}$. This is a probability measure on $G(d, k)$, the Grassmannian of $k$-dimensional linear subspaces of $\mathbb{R}^{d}$.

Let $\mathcal{P}^{d}$ denote the space of (nonempty, compact, convex) polytopes in $\mathbb{R}^{d}$, with the topology induced by the Hausdorff metric.

Theorem 1. Let $k \in\{1, \ldots, d-1\}$. The distribution of the weighted typical $k$-face of the stationary Poisson hyperplane tessellation $X$ is given by

$$
\mathrm{P}\left(Z_{0}^{(k)} \in A\right)=\int_{G(d, k)} \mathrm{P}\left(Z_{0} \cap L \in A\right) \widehat{\mathbb{Q}}_{d-k}(\mathrm{~d} L)
$$

for $A \in \mathcal{B}\left(\mathscr{P}^{d}\right)$.

The weighted typical $k$-face $Z_{0}^{(k)}$ is thus stochastically equivalent to the random polytope $Z_{0} \cap \mathcal{L}$, where $\mathcal{L}$ is a random $k$-dimensional subspace of $\mathbb{R}^{d}$, independent of $\widehat{X}$, with distribution $\widehat{\mathbb{Q}}_{d-k}$.

We point out a consequence in the isotropic case. If $\widehat{X}$ is isotropic (its distribution is invariant under rotations) then there exists a random rotation $\vartheta$ such that $\vartheta Z_{0}^{(k)}$ has the same distribution as the zero cell of a stationary isotropic Poisson hyperplane process in $k$-dimensional Euclidean 
space $\mathbb{R}^{k}$ (considered as a subspace of $\mathbb{R}^{d}$ ). Therefore, in the isotropic case, the study of motion invariant characteristics of the weighted $k$-face is comprised by the study of the zero cell in $\mathbb{R}^{k}$.

New aspects, however, arise in the anisotropic case. The following theorem on sharp inequalities for the expected vertex number extends a result which is known for the zero cell (cf. [11, Theorem 10.4.9]). By $f_{0}(P)$ we denote the number of vertices of a polytope $P$. The constant $\kappa_{k}$ is the $k$-dimensional volume of the $k$-dimensional unit ball.

Theorem 2. Let $k \in\{2, \ldots, d\}$. Then

$$
2^{k} \leq \mathrm{E} f_{0}\left(Z_{0}^{(k)}\right) \leq 2^{-k} k ! \kappa_{k}^{2} .
$$

Equality holds on the left-hand side if and only if $X$ is a parallel mosaic. Equality on the right-hand side holds if $X$ is isotropic.

A parallel mosaic in $\mathbb{R}^{d}$ is a hyperplane tessellation where the hyperplanes belong to $d$ fixed translation classes. Thus, all cells of a parallel mosaic are parallelepipeds. If they are rectangular parallelepipeds, the hyperplane process has been called a cuboid process. Stationary Poisson cuboid processes were studied by Favis [3], [4].

We conjecture that equality on the right-hand side of (1) holds only if $X$ is isotropic with respect to some Euclidean metric on $\mathbb{R}^{d}$.

We point out that, for the typical $k$-face, E $f_{0}\left(Z^{(k)}\right)=2^{k}$ always, even without the Poisson assumption, by a result of J. Mecke (see [11, Theorem 10.3.1]).

\section{Palm distributions and weighted faces}

We introduce the Palm distribution which we employ to give a precise definition of weighted $k$-faces. We adopt and slightly extend the approach of Baumstark and Last [2] and first recall some basic definitions and facts, closely following the presentation and terminology in [11]; in particular, notions and results from Chapters 3, 4, and 10 are used throughout. As there, we identify a simple counting measure $\eta$ with its support and use $\eta(\{x\})=1$ and $x \in \eta$ synonymously. Recall that $G(d, k)$ is the Grassmannian of $k$-dimensional linear subspaces and $A(d, k)$ is the affine Grassmannian of $k$-dimensional flats in $\mathbb{R}^{d}$. In the following we abbreviate $\mathscr{B}:=\mathscr{B}\left(\mathbb{R}^{d}\right), \mathrm{N}_{s}:=\mathrm{N}_{s}(A(d, d-1))$, and $\mathcal{N}_{s}:=\mathcal{N}_{s}(A(d, d-1))$, where $\mathrm{N}_{s}(E)$ is the set of simple counting measures on a space $E$ and $\mathcal{N}_{s}(E)$ is its usual $\sigma$-algebra (see [11, Section 3.1]). We denote by $\mathrm{M}\left(\mathbb{R}^{d}\right)$ the set of locally finite Borel measures on $\mathbb{R}^{d}$ and by $\mathcal{M}\left(\mathbb{R}^{d}\right)$ its usual $\sigma$-algebra. For $0 \leq k<d, \mathscr{H}^{k}$ is the $k$-dimensional Hausdorff measure on $\mathbb{R}^{d}$, but we prefer the notation $\lambda$ for the Lebesgue measure on $\mathbb{R}^{d}$.

Let $\widehat{X}$ be a nondegenerate stationary Poisson hyperplane process in $\mathbb{R}^{d}$, with intensity measure $\widehat{\Theta}$. The latter is a locally finite measure on $A(d, d-1)$. By [11, Theorem 4.4.2 and (4.30)], it has a decomposition such that

$$
\int_{A(d, d-1)} f \mathrm{~d} \widehat{\Theta}=\widehat{\gamma} \int_{S^{d-1}} \int_{-\infty}^{\infty} f\left(u^{\perp}+\tau u\right) \mathrm{d} \tau \widehat{\varphi}(\mathrm{d} u)
$$

for every nonnegative, measurable function $f$ on $A(d, d-1)$, where $\widehat{\gamma}>0$ is the intensity and $\widehat{\varphi}$ is the spherical directional distribution of $\widehat{X}$.

In the introduction we have already defined the induced tessellation $X=X^{(d)}$ and its $k$-face processes $X^{(k)}$, with intensity $\gamma^{(k)}$ and grain distribution $\mathbb{Q}^{(k)}, k=0, \ldots, d$, as well as 
the intersection process $\widehat{X}_{d-k}$ of order $d-k$ with intensity $\widehat{\gamma}_{d-k}$ and directional distribution $\widehat{\mathbb{Q}}_{d-k}$, for $k \in\{0, \ldots, d-1\}$ (recall that the index $d-k$ refers to the number of intersecting hyperplanes and not to the dimension of the flats of $\widehat{X}_{d-k}$ ).

Let $m: \mathrm{N}_{s} \rightarrow \mathrm{M}\left(\mathbb{R}^{d}\right)$ be a measurable mapping satisfying $m(\eta+x)=m(\eta)+x$ for all $\eta \in \mathrm{N}_{s}$ and all $x \in \mathbb{R}^{d}$ (with the usual definition $(\mu+x)(A):=\mu(A-x)$ for a measure $\mu$ if $\mathbb{R}^{d}$ operates as a translation group on its domain). Then $M:=m \circ \widehat{X}$ is a stationary random measure on $\mathbb{R}^{d}$. Let $\gamma_{M}$ denote its intensity. We assume that $0<\gamma_{M}<\infty$ (this will be satisfied in the concrete cases studied below). We introduce the Palm distribution $\mathrm{P}_{M}^{0}$ of the pair $(\widehat{X}, M)$, generalizing the usual procedure via the Campbell measure. For $\widetilde{A} \in \mathscr{B} \otimes \mathcal{N}_{s}$, let

$$
\mu(\widetilde{\mathcal{A}}):=\mathrm{E} \int_{\mathbb{R}^{d}} \mathbf{1}_{\widetilde{\mathcal{A}}}(x, \widehat{X}-x) M(\mathrm{~d} x), \quad \mathcal{C}(\widetilde{\mathcal{A}}):=\mathrm{E} \int_{\mathbb{R}^{d}} \mathbf{1}_{\widetilde{\mathcal{A}}}(x, \widehat{X}) M(\mathrm{~d} x) .
$$

Then $\mu$ and $\mathcal{C}$ are measures. Let $B \in \mathscr{B}$ and $\mathcal{A} \in \mathcal{N}_{s}$. For $y \in \mathbb{R}^{d}$, we obtain, substituting $x$ by $x+y$ and using the stationarity of $\widehat{X}($ and writing $m(\eta)(\cdot)=m(\eta, \cdot))$

$$
\begin{aligned}
\mu((B+y) \times \mathcal{A}) & =\mathrm{E} \int_{\mathbb{R}^{d}} \mathbf{1}_{B+y}(x) \mathbf{1}_{\mathcal{A}}(\widehat{X}-x) m(\widehat{X}, \mathrm{~d} x) \\
& =\mathrm{E} \int_{\mathbb{R}^{d}} \mathbf{1}_{B}(x) \mathbf{1}_{\mathcal{A}}(\widehat{X}-y-x) m(\widehat{X}-y, \mathrm{~d} x) \\
& =\mathrm{E} \int_{\mathbb{R}^{d}} \mathbf{1}_{B}(x) \mathbf{1}_{\mathcal{A}}(\widehat{X}-x) M(\mathrm{~d} x) \\
& =\mu(B \times \mathcal{A}) .
\end{aligned}
$$

Thus, $\mu$ is translation invariant in its first argument. Since it is locally finite, we have $\mu(B \times \mathcal{A})=$ $\alpha(\mathcal{A}) \lambda(B)$ with a finite measure $\alpha$. Furthermore,

$$
\mu\left(B \times \mathrm{N}_{s}\right)=\mathrm{E} \int_{\mathbb{R}^{d}} \mathbf{1}_{B}(x) M(\mathrm{~d} x)=\mathrm{E} M(B)=\gamma_{M} \lambda(B) .
$$

Hence, if we put $\mathrm{P}_{M}^{0}:=\gamma_{M}^{-1} \mu\left(C^{d} \times \cdot\right)$, where $C^{d}:=[0,1]^{d}$ is the unit cube, then $\mathrm{P}_{M}^{0}$ is a probability measure on $\mathrm{N}_{s}$, and

$$
\mu=\gamma_{M} \lambda \otimes \mathrm{P}_{M}^{0}
$$

Thus, by (3),

$$
\gamma_{M} \mathrm{P}_{M}^{0}(\mathcal{A})=\mathrm{E} \int_{\mathbb{R}^{d}} \mathbf{1}_{B}(x) \mathbf{1}_{\mathcal{A}}(\widehat{X}-x) M(\mathrm{~d} x)
$$

for any set $B_{\widetilde{A}} \in \mathcal{B}$ with $\lambda(\underset{\mathcal{A}}{B})=1$. This defines the Palm distribution $\mathrm{P}_{M}^{0}$ of the pair $(\widehat{X}, M)$.

Since $\mathcal{C}(\widetilde{A})=\mu\left(T^{-1} \widetilde{\mathcal{A}}\right)$ for the map $T:(x, \eta) \mapsto(x, \eta+x)$, we see from (3) and (4) that

$$
\mathrm{E}\left[\mathbf{1}_{\mathcal{A}}(\widehat{X}) M(B)\right]=\mathcal{C}(B \times \mathcal{A})=\gamma_{M} \int_{\mathbb{R}^{d}} \mathbf{1}_{B}(x) \mathrm{P}_{M}^{0}(\mathcal{A}-x) \lambda(\mathrm{d} x)
$$

for all $B \in \mathscr{B}$ and $\mathcal{A} \in \mathcal{N}_{S}$. The standard extension procedure gives the refined Campbell theorem for the pair $(\widehat{X}, M)$, namely

$$
\mathrm{E} \int_{\mathbb{R}^{d}} f(x, \widehat{X}) M(\mathrm{~d} x)=\gamma_{M} \int_{\mathbb{R}^{d}} \int_{\mathrm{N}_{s}} f(x, \eta+x) \mathrm{P}_{M}^{0}(\mathrm{~d} \eta) \lambda(\mathrm{d} x)
$$

for every nonnegative, measurable function $f$ on $\mathbb{R}^{d} \times \mathrm{N}_{s}$. 
Now we introduce weighted typical $k$-faces, for $k \in\{1, \ldots, d\}$. If $\eta \in \mathbf{N}_{s}$, the hyperplanes in the support of $\eta$ induce a tessellation of $\mathbb{R}^{d}$ (possibly with unbounded cells), and we denote by $\mathcal{F}_{k}(\eta)$ the set of $k$-faces of this tessellation (so that $\mathcal{F}_{k}(\widehat{X})=X^{(k)}$ ). For $\eta \in \mathrm{N}_{s}$, we define $F_{0}^{k}(\eta)$ as the (necessarily unique) face $F \in \mathcal{F}_{k}(\eta)$ with $0 \in$ relint $F$, if such a face exists, and $F_{0}^{k}(\eta):=\{0\}$ otherwise. Let $Y_{M}$ be a hyperplane process with distribution $\mathrm{P}_{M}^{0}$, and define a random polytope by

$$
Z_{M}^{(k)}:=F_{0}^{k}\left(Y_{M}\right)
$$

In the special cases considered below, $Z_{M}^{(k)}$ is a.s. of dimension $k$. For the distribution of $Z_{M}^{(k)}$, from (5), we obtain

$$
\mathrm{P}\left(Z_{M}^{(k)} \in A\right)=\frac{1}{\gamma_{M}} \mathrm{E} \int_{\mathbb{R}^{d}} \mathbf{1}_{B}(x) \mathbf{1}_{A}\left(F_{0}^{k}(\widehat{X}-x)\right) M(\mathrm{~d} x)
$$

for $A \in \mathscr{B}\left(\mathcal{P}^{d}\right)$ and $B \in \mathscr{B}$ with $\lambda(B)=1$.

For the first example, let $s$ be the centre function defined by the Steiner point; then $s(P) \in$ relint $P$ for all $P \in \mathcal{P}^{d}$. Let $k \in\{1, \ldots, d\}$. For $m$, we take the measure valued map $n_{k}$ defined by

$$
n_{k}(\eta):=\sum_{F \in \mathcal{F}_{k}(\eta)} \delta_{s(F)}, \quad \eta \in \mathrm{N}_{s}
$$

and we write $n_{k} \circ \widehat{X}=: N_{k}$. Then from (7) we obtain, noting that $F_{0}^{k}(\widehat{X}-s(F))=F-s(F)$ for $F \in X^{(k)}$,

$$
\mathrm{P}\left(Z_{N_{k}}^{(k)} \in A\right)=\frac{1}{\gamma^{(k)}} \mathrm{E} \sum_{F \in X^{(k)}} \mathbf{1}_{B}(s(F)) \mathbf{1}_{A}(F-s(F))=\mathbb{Q}^{(k)}(A) .
$$

The last equation follows from [11, Definition (4.6) and Theorem 4.1.3(a)], (cf. also p. 106), with $\varphi(P):=\mathbf{1}_{A}(P-s(P))$. Hence, $Z_{N_{k}}^{(k)}$ is stochastically equivalent to the typical $k$-face $Z^{(k)}$, with respect to the Steiner point as the centre function.

Let $m$ and $M$ be general as before, and let $h$ be a translation invariant, nonnegative, measurable function on $\mathcal{P}^{d}$. (The translation invariance is not a severe restriction, since from any $h$ we can pass to the translation invariant function $P \mapsto h(P-s(P))$.) Slightly extending a remark in [2], we apply [11, Theorem 3.4.5] (Neveu's exchange formula) to the probability space $\left(\mathrm{N}_{s}, \mathcal{N}_{s}, \mathrm{P}_{\widehat{X}}\right)$, the random measures $m, n_{k}$, and the function $f(y, \eta):=h\left(F_{0}^{k}(\eta)\right) \mathbf{1}_{F_{0}^{k}(\eta)}(-y)$. (Observe that $\mathrm{P}_{M}^{0}$ is now equal to the Palm distribution of the random measure $m$, in the sense of $[11$, p. 80].) We obtain

$$
\gamma_{M} \int_{\mathrm{N}_{s}} \sum_{F \in \mathcal{F}_{k}(\eta), 0 \in F} h(F) \mathrm{P}_{M}^{0}(\mathrm{~d} \eta)=\gamma^{(k)} \int_{\mathrm{N}_{s}} h\left(F_{0}^{k}(\eta)\right) m(\eta)\left(F_{0}^{k}(\eta)\right) \mathrm{P}_{N_{k}}^{0}(\mathrm{~d} \eta) .
$$

For the second example, we take (denoting by ' $L$ ' the restriction of a measure)

$$
m_{k}(\eta):=\sum_{F \in \mathcal{F}_{k}(\eta)} \mathscr{H}^{k}\left\llcorner\text { relint } F, \quad \eta \in \mathrm{N}_{s},\right.
$$

and set $m_{k} \circ \widehat{X}=: M_{k}$. We simplify the notation by writing

$$
\mathrm{P}_{M_{k}}^{0}=: \mathrm{P}_{k}^{0}, \quad Z_{M_{k}}^{(k)}=: Z_{0}^{(k)} .
$$


Note that

$$
\gamma_{M_{k}}=\widehat{\gamma}_{d-k}
$$

In fact, for $B \in \mathscr{B}$, we have $\gamma_{M_{k}} \lambda(B)=\mathrm{E} M_{k}(B)=\widehat{\gamma}_{d-k} \lambda(B)$ by [11, Theorem 4.4.3], applied to $\widehat{X}_{d-k}$.

Relation (8) can now be written in the form

$$
\mathrm{E} h\left(Z_{0}^{(k)}\right)=\frac{1}{\mathrm{E} V_{k}\left(Z^{(k)}\right)} \mathrm{E}\left[h\left(Z^{(k)}\right) V_{k}\left(Z^{(k)}\right)\right]
$$

where $V_{k}(K)$, for a $k$-dimensional convex set $K$, is its $k$-dimensional volume. This shows that the distribution of $Z_{0}^{(k)}$ is, up to translations, the volume-weighted distribution of the typical $k$-face $Z^{(k)}$. For this reason, the random polytope $Z_{0}^{(k)}$ is called the weighted typical $k$-face.

The connection with the heuristic explanation in the introduction can be seen from the relation

$$
\mathrm{P}\left(Z_{0}^{(k)}-s\left(Z_{0}^{(k)}\right) \in A\right)=\lim _{r \rightarrow \infty} \frac{\mathrm{E} \sum_{F \in X^{(k)}, F \subset r W} \mathbf{1}_{A}(F-s(F)) V_{k}(F)}{\mathrm{E} \sum_{F \in X^{(k)}, F \subset r W} V_{k}(F)},
$$

valid for $A \in \mathscr{B}\left(\mathcal{P}^{d}\right)$ and any compact convex set $W \subset \mathbb{R}^{d}$ with $\lambda(W)>0$. Relation (11) follows from [11, Theorem 4.1.3(b)], together with (10).

In the proof of Theorem 1, we use a Slivnyak-type result, which we prove now. We need the following transformation formula. Note that, for $u \in S^{d-1}$ and $\tau \in \mathbb{R}$,

$$
u^{\perp}+\tau u=H(u, \tau):=\left\{x \in \mathbb{R}^{d}:\langle x, u\rangle=\tau\right\}
$$

is a hyperplane. Let $k \in\{1, \ldots, d\}$. If $f$ is a nonnegative measurable function on $A(d, d-1)^{k}$, then, for linearly independent vectors $u_{1}, \ldots, u_{k} \in S^{d-1}$ with $U:=\operatorname{lin}\left\{u_{1}, \ldots, u_{k}\right\}$,

$$
\begin{aligned}
\int_{-\infty}^{\infty} & \cdots \int_{-\infty}^{\infty} f\left(H\left(u_{1}, \tau_{1}\right), \ldots, H\left(u_{k}, \tau_{k}\right)\right) \mathrm{d} \tau_{1} \cdots \mathrm{d} \tau_{k} \\
& =\nabla_{k}\left(u_{1}, \ldots, u_{k}\right) \int_{U} f\left(u_{1}^{\perp}+z, \ldots, u_{k}^{\perp}+z\right) \mathscr{H}^{k}(\mathrm{~d} z) .
\end{aligned}
$$

Here $\nabla_{k}\left(u_{1}, \ldots, u_{k}\right)$ denotes the $k$-dimensional volume of the parallelepiped spanned by $u_{1}, \ldots, u_{k}$. For the proof of (13), we write

$$
f\left(H\left(u_{1}, \tau_{1}\right), \ldots, H\left(u_{k}, \tau_{k}\right)\right)=: g\left(\tau_{1}, \ldots, \tau_{k}\right)
$$

and define $T\left(\tau_{1}, \ldots, \tau_{k}\right):=\left(z_{1}, \ldots, z_{k}\right)=z$, where $z$ is the unique point in the intersection $H\left(u_{1}, \tau_{1}\right) \cap \cdots \cap H\left(u_{k}, \tau_{k}\right) \cap U$. The map $T$ is injective; its inverse is given by $T^{-1}(z)=$ $\left(\left\langle z, u_{1}\right\rangle, \ldots,\left\langle z, u_{k}\right\rangle\right)$ and has Jacobian $\nabla_{k}\left(u_{1}, \ldots, u_{k}\right)$. Hence, the transformation formula for multiple integrals gives

$$
\int g\left(\tau_{1}, \ldots, \tau_{k}\right) \mathrm{d} \tau_{1} \cdots \mathrm{d} \tau_{k}=\int g\left(T^{-1}(z)\right) \nabla_{k}\left(u_{1}, \ldots, u_{k}\right) \lambda(\mathrm{d} z),
$$

which together with $g\left(T^{-1}(z)\right)=g\left(\left\langle z, u_{1}\right\rangle, \ldots,\left\langle z, u_{k}\right\rangle\right)$ and (12) yields (13).

We define a measure $\phi_{k}$ on $G(d, d-1)^{k}$ by

$$
\phi_{k}(A):=\frac{\widehat{\gamma}^{k}}{k ! \widehat{\gamma}_{k}} \int_{\left(S^{d-1}\right)^{k}} \mathbf{1}_{A}\left(u_{1}^{\perp}, \ldots, u_{k}^{\perp}\right) \nabla_{k}\left(u_{1}, \ldots, u_{k}\right) \widehat{\varphi}^{k}\left(\mathrm{~d}\left(u_{1}, \ldots, u_{k}\right)\right)
$$

for $A \in \mathcal{B}\left(G(d, d-1)^{k}\right)$. 
Since we identify simple counting measures with their supports, $\widehat{X} \cup\left\{H_{1}, \ldots, H_{k}\right\}$ below also denotes the counting measure $\widehat{X}+\delta_{H_{1}}+\cdots+\delta_{H_{k}}$, which is a random element of $\mathrm{N}_{s}$. In the next theorem we interchange, for convenience, the roles of $k$ and $d-k$.

Theorem 3. Suppose that $\widehat{X}$ is a nondegenerate stationary Poisson hyperplane process. For $k=1, \ldots, d$ and $\mathcal{A} \in \mathcal{N}_{s}$,

$$
\mathrm{P}_{d-k}^{0}(\mathcal{A})=\int_{G(d, d-1)^{k}} \mathrm{P}\left(\widehat{X} \cup\left\{H_{1}, \ldots, H_{k}\right\} \in \mathcal{A}\right) \phi_{k}\left(\mathrm{~d}\left(H_{1}, \ldots, H_{k}\right)\right) .
$$

Proof. Let $B \in \mathscr{B}$ and $\mathcal{A} \in \mathcal{N}_{s}$. In the following we use, in this order, the refined Campbell theorem (6) together with (9), the definition of the random measure $M_{d-k}$, the Slivnyak-Mecke formula (Corollary 3.2.3 of [11]), and decomposition (2) of the intensity measure $\widehat{\Theta}$. Since $\widehat{X}$ is a stationary Poisson hyperplane process, a.s. any $k$ of its hyperplanes intersect either in the empty set or in a $(d-k)$-flat $[11$, p. 134]. We obtain

$$
\begin{aligned}
\lambda(B) \mathrm{P}_{d-k}^{0}(\mathcal{A}) & \\
= & \int_{\mathbb{R}^{d}} \int_{\mathrm{N}_{s}} \mathbf{1}_{B}(x) \mathbf{1}_{\mathcal{A}}(\eta) \mathrm{P}_{d-k}^{0}(\mathrm{~d} \eta) \lambda(\mathrm{d} x) \\
= & \frac{1}{\widehat{\gamma}_{k}} \mathrm{E} \int_{\mathbb{R}^{d}} \mathbf{1}_{B}(x) \mathbf{1}_{\mathcal{A}}(\widehat{X}-x) M_{d-k}(\mathrm{~d} x) \\
= & \frac{1}{k ! \widehat{\gamma}_{k}} \mathrm{E} \sum_{\left(H_{1}, \ldots, H_{k}\right) \in \widehat{X}_{\neq}^{k}} \int_{H_{1} \cap \cdots \cap H_{k}} \mathbf{1}_{B}(x) \mathbf{1}_{\mathcal{A}}(\widehat{X}-x) \mathscr{H}^{d-k}(\mathrm{~d} x) \\
= & \frac{1}{k ! \widehat{\gamma}_{k}} \int_{A(d, d-1)^{k}} \mathrm{E} \int_{H_{1} \cap \cdots \cap H_{k}} \mathbf{1}_{B}(x) \mathbf{1}_{\mathcal{A}}\left(\left(\widehat{X} \cup\left\{H_{1}, \ldots, H_{k}\right\}\right)-x\right) \mathscr{H}^{d-k}(\mathrm{~d} x) \\
= & \frac{\widehat{\gamma}^{k}}{k ! \widehat{\gamma}_{k}} \int_{\left(S^{d-1}\right)^{k}} \int_{-\infty}^{\infty} \ldots \int_{-\infty}^{\infty} \mathrm{E} \int_{H\left(u_{1}, \tau_{1}\right) \cap \cdots \cap H\left(u_{k}, \tau_{k}\right)} \mathbf{1}_{B}(x) \\
& \quad \times \mathbf{1}_{\mathcal{A}}\left(\left(\widehat{X} \cup\left\{H\left(H_{1}, \ldots, \tau_{1}\right), \ldots, H\left(u_{k}, \tau_{k}\right)\right\}\right)-x\right) \mathscr{H}^{d-k}(\mathrm{~d} x) \mathrm{d} \tau_{1} \cdots \mathrm{d} \tau_{k} \\
& \quad \times \widehat{\varphi}^{k}\left(\mathrm{~d}\left(u_{1}, \ldots, u_{k}\right)\right) .
\end{aligned}
$$

By the transformation formula (13), we have

$$
\begin{aligned}
& \int_{-\infty}^{\infty} \cdots \int_{-\infty}^{\infty} \mathrm{E} \int_{H\left(u_{1}, \tau_{1}\right) \cap \ldots \cap H\left(u_{k}, \tau_{k}\right)} \mathbf{1}_{B}(x) \mathbf{1}_{\mathcal{A}}\left(\left(\widehat{X} \cup\left\{H\left(u_{1}, \tau_{1}\right), \ldots, H\left(u_{k}, \tau_{k}\right)\right\}\right)-x\right) \\
& \times \mathscr{H}^{d-k}(\mathrm{~d} x) \mathrm{d} \tau_{1} \cdots \mathrm{d} \tau_{k} \\
& =\nabla_{k}\left(u_{1}, \ldots, u_{k}\right) \int_{\operatorname{lin}\left\{u_{1}, \ldots, u_{k}\right\}} \int_{\left(u_{1}^{\perp} \cap \ldots \cap u_{k}^{\perp}\right)+z} \mathbf{1}_{B}(x) \mathrm{E} \mathbf{1}_{\mathcal{A}}\left(\left(\widehat{X} \cup\left\{u_{1}^{\perp}+z, \ldots, u_{k}^{\perp}+z\right\}\right)-x\right) \\
& \times \mathscr{H}^{d-k}(\mathrm{~d} x) \mathscr{H}^{k}(\mathrm{~d} z) \\
& =\nabla_{k}\left(u_{1}, \ldots, u_{k}\right) \int_{\operatorname{lin}\left\{u_{1}, \ldots, u_{k}\right\}} \int_{\left(u_{1}^{\perp} \cap \ldots \cap u_{k}^{\perp}\right)+z} \mathbf{1}_{B}(x) \mathrm{E} \mathbf{1}_{\mathcal{A}}\left((\widehat{X}-x) \cup\left\{u_{1}^{\perp}, \ldots, u_{k}^{\perp}\right\}\right) \\
& \times \mathscr{H}^{d-k}(\mathrm{~d} x) \mathscr{H}^{k}(\mathrm{~d} z) \\
& =\nabla_{k}\left(u_{1}, \ldots, u_{k}\right) \lambda(B) \mathrm{P}\left(\widehat{X} \cup\left\{u_{1}^{\perp}, \ldots, u_{k}^{\perp}\right\} \in \mathcal{A}\right) \text {. }
\end{aligned}
$$


Above, we have used the facts that $x \in u^{\perp}+z$ implies that $u^{\perp}+z-x=u^{\perp}$ and that $\widehat{X}$ is stationary. In view of (14), the proof of Theorem 3 is complete.

\section{Proof of Theorem 1}

The weighted typical $k$-face of the tessellation $X$ was defined (up to stochastic equivalence) by $Z_{0}^{(k)}=F_{0}^{k}\left(Y_{M_{k}}\right)$, where $Y_{M_{k}}$ is a hyperplane process with distribution $\mathrm{P}_{M_{k}}^{0}=\mathrm{P}_{k}^{0}$. (Note that $\mathrm{P}_{d}^{0}$ is the distribution of $X$ and, hence, $Z_{0}^{(d)}$ is stochastically equivalent to the zero cell $Z_{0}$.) It follows from Theorem 3 (with $k$ and $d-k$ interchanged again) that, for any nonnegative measurable function $g$ on $\mathrm{N}_{s}$, we have

$$
\mathrm{E} g\left(Y_{M_{k}}\right)=\int_{G(d, d-1)^{d-k}} \mathrm{E} g\left(\widehat{X} \cup\left\{H_{1}, \ldots, H_{d-k}\right\}\right) \phi_{d-k}\left(\mathrm{~d}\left(H_{1}, \ldots, H_{d-k}\right)\right) .
$$

Let $f$ be a nonnegative measurable function on the space of $k$-dimensional polytopes. From (15) we obtain

$$
\text { E } \begin{aligned}
f\left(Z_{0}^{(k)}\right) & =\int_{G(d, d-1)^{d-k}} \operatorname{E~} f\left(F_{0}^{k}\left(\widehat{X} \cup\left\{H_{1}, \ldots, H_{d-k}\right\}\right)\right) \phi_{d-k}\left(\mathrm{~d}\left(H_{1}, \ldots, H_{d-k}\right)\right) \\
& =\int_{G(d, d-1)^{d-k}} \operatorname{E~} f\left(Z_{0} \cap H_{1} \cap \cdots \cap H_{d-k}\right) \phi_{d-k}\left(\mathrm{~d}\left(H_{1}, \ldots, H_{d-k}\right)\right),
\end{aligned}
$$

by the definition of $F_{0}^{k}$, and, hence, for $A \in \mathcal{B}\left(\mathcal{P}^{d}\right)$,

$$
\mathrm{P}\left(Z_{0}^{(k)} \in A\right)=\int_{G(d, d-1)^{d-k}} \mathrm{P}\left(Z_{0} \cap H_{1} \cap \cdots \cap H_{d-k} \in A\right) \phi_{d-k}\left(\mathrm{~d}\left(H_{1}, \ldots, H_{d-k}\right)\right) .
$$

According to [11, Theorem 4.4.8] and (14) we have

$$
\widehat{\mathbb{Q}}_{d-k}(A)=\int_{G(d, d-1)^{d-k}} \mathbf{1}_{A}\left(H_{1} \cap \cdots \cap H_{d-k}\right) \phi_{d-k}\left(\mathrm{~d}\left(H_{1}, \ldots, H_{d-k}\right)\right)
$$

for $A \in \mathscr{B}(G(d, k))$. This together with (16) proves Theorem 1 .

After the formulation of Theorem 1, we made a remark about the isotropic case, which we now substantiate. Suppose that $\widehat{X}$ is isotropic. Let $S \in G(d, k)$ be a fixed subspace. We can choose a measurable mapping $D: G(d, k) \rightarrow S O_{d}$, where $S O_{d}$ is the rotation group, with the property that $D(L) L=S$ for all $L \in G(d, k)$. Since the distribution of $Z_{0}$ is rotation invariant, we obtain, for $A \in \mathscr{B}\left(\mathscr{P}^{d}\right)$,

$$
\begin{aligned}
\mathrm{P}\left(D\left(\operatorname{lin} Z_{0}^{(k)}\right) Z_{0}^{(k)} \in A\right) & =\int_{G(d, k)} \mathrm{P}\left(D\left(\operatorname{lin}\left(Z_{0} \cap L\right)\right)\left(Z_{0} \cap L\right) \in A\right) \widehat{\mathbb{Q}}_{d-k}(\mathrm{~d} L) \\
& =\int_{G(d, k)} \mathrm{P}\left(D(L) Z_{0} \cap S \in A\right) \widehat{\mathbb{Q}}_{d-k}(\mathrm{~d} L) \\
& =\mathrm{P}\left(Z_{0} \cap S \in A\right) \\
& =\mathrm{P}\left(Z_{0}(\widehat{X} \cap S) \in A\right),
\end{aligned}
$$

where $Z_{0}(\widehat{X} \cap S)$ is the zero cell of the section process $\widehat{X} \cap S$. The latter is a stationary and isotropic Poisson hyperplane process in the $k$-dimensional space $S$. Thus, if $\widehat{X}$ is isotropic then there is a random rotation $\vartheta$ (namely, $\vartheta(\omega)=D\left(\operatorname{lin} Z_{0}^{(k)}(\omega)\right)$ for $\omega \in \Omega$ ) such that $\vartheta Z_{0}^{(k)}$ has the same distribution as the zero cell of a stationary, isotropic Poisson hyperplane process in $\mathbb{R}^{k}$. 


\section{Proof of Theorem 2}

For the proof of Theorem 2, we note that Theorem 1 implies that

$$
\text { E } f\left(Z_{0}^{(k)}\right)=\int_{G(d, k)} \operatorname{E~} f\left(Z_{0} \cap L\right) \widehat{\mathbb{Q}}_{d-k}(\mathrm{~d} L)
$$

for every nonnegative, measurable function $f$ on $\mathcal{P}^{d}$. The intersection $Z_{0} \cap L$ is the zero cell of the intersection process $\widehat{X} \cap L$, denoted by $Z_{0}(\widehat{X} \cap L)$; hence, for the vertex number $f_{0}$, we obtain

$$
\mathrm{E} f_{0}\left(Z_{0}^{(k)}\right)=\int_{G(d, k)} \mathrm{E} f_{0}\left(Z_{0}(\widehat{X} \cap L)\right) \widehat{\mathbb{Q}}_{d-k}(\mathrm{~d} L)
$$

We recall the definition of the associated zonoid of the stationary hyperplane process $\widehat{X}$. This is the convex body $\Pi_{\widehat{X}}$ with support function

$$
h\left(\Pi_{\widehat{X}}, u\right)=\frac{\widehat{\gamma}}{2} \int_{S^{d-1}}|\langle u, v\rangle| \widehat{\varphi}(\mathrm{d} v)
$$

for $u \in \mathbb{R}^{d}$; see [11, Equation (4.59)]. The section process $\widehat{X} \cap L$ is a stationary Poisson hyperplane process with respect to $L$. Its associated zonoid is given by

$$
\Pi_{\widehat{X} \cap L}=\Pi_{\widehat{X}} \mid L,
$$

where $\cdot \mid L$ denotes the orthogonal projection to $L$; see [11, Equation (4.61)]. From [11, Theorem 10.4.9], applied in $L$, we deduce that

$$
\text { E } f_{0}\left(Z_{0}(\widehat{X} \cap L)\right)=2^{-k} k ! V_{k}\left(\Pi_{\widehat{X}} \mid L\right) V_{k}\left(\left(\Pi_{\widehat{X}} \mid L\right)^{o}\right) \text {. }
$$

Here, $V_{k}$ denotes the $k$-dimensional volume of a convex body and $K^{o}$ is the polar body of $K$ in the subspace $L$. Hence, we arrive at

$$
\text { E } f_{0}\left(Z_{0}^{(k)}\right)=2^{-k} k ! \int_{G(d, k)} V_{k}\left(\Pi_{\widehat{X}} \mid L\right) V_{k}\left(\left(\Pi_{\widehat{X}} \mid L\right)^{o}\right) \widehat{\mathbb{Q}}_{d-k}(\mathrm{~d} L) .
$$

For a $k$-dimensional zonoid $K$ with centre 0 , the inequalities

$$
\frac{4^{k}}{k !} \leq V_{k}(K) V_{k}\left(K^{o}\right) \leq \kappa_{k}^{2}
$$

are valid. The right-hand side is the Blaschke-Santaló inequality, and the left-hand side, for zonoids, is Reisner's inequality (references are in [11, Chapter 14]). Equality on the left holds if and only if $K$ is a parallelepiped, and equality on the right holds if and only if $K$ is an ellipsoid. Applying (18) to $\Pi_{\widehat{X}} \mid L$ in each $L \in G(d, k)$, we obtain from (17) the inequalities of Theorem 2.

Equality holds in the left- and right-hand inequalities of (1) if and only if equality holds in the left- and right-hand inequalities, respectively, of

$$
\frac{4^{k}}{k !} \leq V_{k}\left(\Pi_{\widehat{X}} \mid L\right) V_{k}\left(\left(\Pi_{\widehat{X}} \mid L\right)^{o}\right) \leq \kappa_{k}^{2}
$$

for all subspaces $L$ in the support of the measure $\widehat{\mathbb{Q}}_{d-k}$. 
Suppose first that $X$ and, therefore, $\widehat{X}$ is isotropic. Then $\widehat{\varphi}$ is rotation invariant and $\Pi_{\widehat{X}}$ is a ball. Hence, for each $L \in G(d, k)$, the projection $\Pi_{\widehat{X}} \mid L$ is a ball, and equality holds on the right-hand side of (19). Thus, equality holds on the right-hand side of (1). This is also true if the associated zonoid $\Pi_{\widehat{X}}$ is an ellipsoid. We have not been able to decide whether this is the only equality case for the right-hand side of (1).

Now suppose that $X$ is a parallel mosaic. Then there are $d$ linearly independent vectors $e_{1}, \ldots, e_{d} \in S^{d-1}$ such that the spherical directional distribution $\widehat{\varphi}$ is concentrated in $\left\{ \pm e_{1}, \ldots, \pm e_{d}\right\}$. The associated zonoid $\Pi_{\widehat{X}}$ is the sum of segments parallel to $e_{1}, \ldots, e_{d}$ and, hence, is a parallelepiped with edges in these directions. Let $L$ be in the support of the measure $\widehat{\mathbb{Q}}_{d-k}$. Then, since $\widehat{\varphi}$ is discrete, $L=u_{1}^{\perp} \cap \cdots \cap u_{d-k}^{\perp}$ with linearly independent vectors $u_{1}, \ldots, u_{d-k}$ in the support of $\widehat{\varphi}$, say $\left(u_{1}, \ldots, u_{d-k}\right)=\left(e_{1}, \ldots, e_{d-k}\right)$; thus, $L=$ $\operatorname{lin}\left\{e_{1}, \ldots, e_{d-k}\right\}^{\perp}$. We have $e_{i} \mid L=0$ for $i=1, \ldots, d-k$; hence, the projection $\Pi_{\widehat{X}} \mid L$ is a parallelepiped with edges parallel to the projections of $e_{d-k+1}, \ldots, e_{d}$. Therefore, equality holds on the left-hand side of (1).

Conversely, suppose that equality holds on the left-hand side of (1), for some $k \geq 2$. Let $u_{1}, \ldots, u_{d-k}$ be linearly independent vectors in the support of the measure $\widehat{\varphi}$. Then $L:=$ $u_{1}^{\perp} \cap \cdots \cap u_{d-k}^{\perp}$ is in the support of $\widehat{\mathbb{Q}}_{d-k}$. Therefore, equality holds on the left-hand side of (19); hence, the orthogonal projection of the associated zonoid $\Pi_{\widehat{X}}$ to the subspace $u_{1}^{\perp} \cap \cdots \cap u_{d-k}^{\perp}$ is a parallelepiped. By the lemma proved below, it follows that $\Pi_{\widehat{X}}$ is itself a parallelepiped. This implies that $X$ is a parallel mosaic.

We prove the lemma that was needed in the proof of Theorem 2. This requires some knowledge about projections of zonoids.

Let $K$ be a zonoid with generating measure $\rho$, that is,

$$
h(K, x)=\int_{S^{d-1}}|\langle x, v\rangle| \rho(\mathrm{d} v), \quad x \in \mathbb{R}^{d},
$$

where $\rho$ is an even finite measure on $S^{d-1}$. We assume that $\operatorname{dim} K=d$. Then $\rho$ is not concentrated on a great subsphere. It is well known that the measure $\rho$ is uniquely determined by $h(K, \cdot)$. In particular, if $K$ is a parallelepiped then $\rho$ is concentrated in $d$ pairs of antipodal points.

For $e \in S^{d-1}$, write $S_{e}:=e^{\perp} \cap S^{d-1}$. Every vector $x \in S^{d-1} \backslash\{e,-e\}$ has a unique representation $x=t e+\sqrt{1-t^{2}} x_{e}$ with $t \in(-1,1)$ and $x_{e} \in S_{e}$. Define the measure $\tilde{\rho}$ on $S^{d-1}$ by

$$
\widetilde{\rho}(A):=\int_{A}\left|\left\langle x, x_{e}\right\rangle\right| \rho(\mathrm{d} x)
$$

for $A \in \mathcal{B}\left(S^{d-1}\right)$. The mapping $\operatorname{proj}_{S_{e}}: x \mapsto x_{e}$ is defined $\widetilde{\rho}$-almost everywhere on $S^{d-1}$ and is measurable. Let $\rho_{e}$ be the image measure of $\tilde{\rho}$ under $\operatorname{proj}_{S_{e}}$. Then $\rho_{e}$ is the generating measure of the zonoid $K \mid e^{\perp}$ (see [12]).

Lemma 1. Let $d \geq 3$ and $k \in\{1, \ldots, d-2\}$. Let $K$ be represented by (20) and suppose that, for any $k$ linearly independent vectors $v_{1}, \ldots, v_{k}$ in $\operatorname{supp} \rho$, the orthogonal projection $K \mid v_{1}^{\perp} \cap \cdots \cap v_{k}^{\perp}$ is a parallelepiped. Then $K$ itself is a parallelepiped.

Proof. First let $k=1$. Let $e \in \operatorname{supp} \rho$. Then $K \mid e^{\perp}$ is a parallelepiped. Hence, there are linearly independent vectors $u_{1}, \ldots, u_{d-1} \in S_{e}$ such that the generating measure $\rho_{e}$ of $K \mid e^{\perp}$ is concentrated in $\left\{ \pm u_{1}, \ldots, \pm u_{d-1}\right\}$. From the preceding description of this generating measure, it follows that the support of the measure $\rho$ is contained in $S_{e, u_{1}} \cup \cdots \cup S_{e, u_{d-1}}$, where $S_{e, u_{i}}:=\operatorname{lin}\left\{e, u_{i}\right\} \cap S^{d-1}$, and that $\rho\left(S_{e, u_{i}} \backslash\{e,-e\}\right)>0$ for $i=1, \ldots, d-1$. 
We can choose a vector $e^{\prime} \in(\operatorname{supp} \rho) \cap\left(S_{e, u_{1}} \backslash\{e,-e\}\right)$. By the same argument as above, there are linearly independent vectors $u_{1}^{\prime}, \ldots, u_{d-1}^{\prime} \in S_{e^{\prime}}$ such that $\rho$ is concentrated on $S_{e^{\prime}, u_{1}^{\prime}} \cup \cdots \cup S_{e^{\prime}, u_{d-1}^{\prime}}$ and that $\rho\left(S_{e^{\prime}, u_{i}^{\prime}} \backslash\left\{e^{\prime},-e^{\prime}\right\}\right)>0$ for $i=1, \ldots, d-1$. One of the sets $S_{e^{\prime}, u_{1}^{\prime}}, \ldots, S_{e^{\prime}, u_{d-1}^{\prime}}$ must coincide with $S_{e, u_{1}}$, since otherwise $e$ would not be in the support of $\rho$; say $S_{e^{\prime}, u_{1}^{\prime}}=S_{e, u_{1}}$. This implies that $u_{1}^{\prime} \in \operatorname{lin}\left\{e, e^{\prime}\right\}$. Moreover, each of the sets $S_{e^{\prime}, u_{2}^{\prime}}, \ldots, S_{e^{\prime}, u_{d-1}^{\prime}}$ must meet one of the sets $S_{e, u_{2}}, \ldots, S_{e, u_{d-1}}$. Suppose, for example, that $S_{e^{\prime}, u_{2}^{\prime}}$ and $S_{e^{\prime}, u_{3}^{\prime}}$ both meet $S_{e, u_{p}}$, with $p \in\{2, \ldots, d-1\}$. If $x \in S_{e^{\prime}, u_{2}^{\prime}} \cap S_{e, u_{p}}$ then $u_{2}^{\prime} \in \operatorname{lin}\left\{e^{\prime}, x\right\}$ and $x \in \operatorname{lin}\left\{e, u_{p}\right\}$; hence, $u_{2}^{\prime} \in \operatorname{lin}\left\{e, e^{\prime}, u_{p}\right\}$. Similarly, $u_{3}^{\prime} \in \operatorname{lin}\left\{e, e^{\prime}, u_{p}\right\}$. It follows that $u_{1}^{\prime}, u_{2}^{\prime}, u_{3}^{\prime} \in \operatorname{lin}\left\{e, e^{\prime}, u_{p}\right\}$. The latter subspace intersects $\left(e^{\prime}\right)^{\perp}$ in a twodimensional subspace. Thus, $u_{1}^{\prime}, u_{2}^{\prime}$, and $u_{3}^{\prime}$ are linearly dependent. This contradiction shows that different sets $S_{e^{\prime}, u_{i}^{\prime}}$ must meet different sets $S_{e, u_{k}}$. After renumbering, we can assume that $S_{e^{\prime}, u_{i}^{\prime}} \cap S_{e, u_{i}}=\left\{v_{i},-v_{i}\right\}$ for $i=2, \ldots, d-1$, and that $S_{e^{\prime}, u_{i}^{\prime}} \cap S_{e, u_{j}}=\varnothing$ for $i \neq j$. Now it is clear that $\rho$ is concentrated in $\left\{ \pm e, \pm e^{\prime}, \pm v_{2}, \ldots, \pm v_{d-1}\right\}$ and, hence, that $K$ is a parallelepiped.

In particular, we have proved the lemma for $d=3$. Now let $d \geq 4$ and suppose that the assertion is true in smaller dimensions. Choose $e \in \operatorname{supp} \rho$. As before, $\rho_{e}$ is the generating measure of the zonoid $K \mid e^{\perp}$.

Let $k \in\{1, \ldots, d-3\}$. Let $u_{1}, \ldots, u_{k}$ be linearly independent vectors in the support of $\rho_{e}$. For $i=1, \ldots, k$, we have $\rho\left(S_{e, u_{i}} \backslash\{e,-e\}\right)>0$; hence, there exists a vector $v_{i} \in$ $S_{e, u_{i}} \cap \operatorname{supp} \rho$ with $v_{i} \neq \pm e$. The vectors $e, v_{1}, \ldots, v_{k}$ are linearly independent. By assumption, the projection $K \mid e^{\perp} \cap v_{1}^{\perp} \cap \cdots \cap v_{k}^{\perp}$ is a parallelepiped. We have $\operatorname{lin}\left\{e, v_{1}, \ldots, v_{k}\right\}=$ $\operatorname{lin}\left\{e, u_{1}, \ldots, u_{k}\right\}$; hence, $K \mid e^{\perp} \cap u_{1}^{\perp} \cap \cdots \cap u_{k}^{\perp}$ is a parallelepiped. Therefore, $\left(K \mid e^{\perp}\right) \mid u_{1}^{\perp} \cap$ $\cdots \cap u_{k}^{\perp}$ is a parallelepiped. Since this is true for any linearly independent vectors $u_{1}, \ldots, u_{k}$ in the support of $\rho_{e}$, it follows from the induction hypothesis that $K \mid e^{\perp}$ is a parallelepiped. Since this holds for each $e \in \operatorname{supp} \rho$, the already settled case $k=1$ shows that $K$ is a parallelepiped. This completes the proof.

To settle the equality case for the right-hand side of (1), we would need a counterpart to Lemma 1, with 'a parallelepiped' replaced by 'an ellipsoid' (twice).

\section{Remark on characteristics of the zero cell}

We prove a formula for the expected number of $(d-k)$-faces of the zero cell, namely

$$
\mathrm{E} f_{d-k}\left(Z_{0}\right)=\widehat{\gamma}_{k} \int_{G(d, d-k)} \mathrm{E} V_{k}\left(Z_{0} \mid L^{\perp}\right) \widehat{\mathbb{Q}}_{k}(\mathrm{~d} L)
$$

Recall that, for a $k$-dimensional convex body $K$, we denote by $V_{k}(K)=\mathscr{H}^{k}(K)$ its $k$-dimensional volume.

For the proof of (21), we note that, a.s., each $(d-k)$-face of $Z_{0}$ is the intersection of $Z_{0}$ with precisely $k$ hyperplanes of $\widehat{X}$, and any intersection of $k$ different hyperplanes of $\widehat{X}$ a.s. intersects $Z_{0}$ either in a $(d-k)$-face or in the empty set. Let $\chi$ denote the Euler characteristic. As in the proof of Theorem 3, we use the Slivnyak-Mecke formula, the decomposition of the 
intensity measure $\widehat{\Theta}$ of $\widehat{X}$, and the transformation formula (13). Thus, we obtain

$$
\begin{aligned}
\mathrm{E} f_{d-k}\left(Z_{0}\right) & \\
= & \frac{1}{k !} \mathrm{E} \sum_{\left(H_{1}, \ldots, H_{k}\right) \in \widehat{X}_{\neq}^{k}} \chi\left(Z_{0} \cap H_{1} \cap \cdots \cap H_{k}\right) \\
= & \frac{1}{k !} \int_{A(d, d-1)^{k}} \mathrm{E} \chi\left(Z_{0} \cap H_{1} \cap \cdots \cap H_{k}\right) \widehat{\Theta}^{k}\left(\mathrm{~d}\left(H_{1}, \ldots, H_{k}\right)\right) \\
= & \frac{\widehat{\gamma}^{k}}{k !} \int_{\left(S^{d-1}\right)^{k}} \int_{-\infty}^{\infty} \cdots \int_{-\infty}^{\infty} \mathrm{E} \chi\left(Z_{0} \cap H\left(u_{1}, \tau_{1}\right) \cap \cdots \cap H\left(u_{k}, \tau_{k}\right)\right) \\
= & \frac{\widehat{\gamma}^{k}}{k !} \int_{\left(S^{d-1}\right)^{k}} \mathrm{E} V_{k}\left(Z_{0} \mid \operatorname{lin}\left\{u_{1}, \ldots, u_{k}\right\}\right) \nabla_{k}\left(u_{1}, \ldots, u_{k}\right) \widehat{\varphi}^{k}\left(\mathrm{~d}\left(u_{1}, \ldots, u_{k}\right)\right) \\
= & \widehat{\gamma}_{k} \int_{G(d, d-k)} \mathrm{E} V_{k}\left(Z_{0} \mid L^{\perp}\right) \widehat{\mathbb{Q}}_{k}(\mathrm{~d} L) .
\end{aligned}
$$

This proves (21).

Suppose now that $\widehat{X}$ is isotropic. Then $\vartheta^{-1} Z_{0}$ has the same distribution as $Z_{0}$, for every $\vartheta$ in the rotation group $S O_{d}$. Let $v$ denote the invariant probability measure on $S O_{d}$. Using an integral-geometric formula ([11, Equation (5.8)]), we conclude that

$$
\begin{aligned}
\mathrm{E} V_{k}\left(Z_{0} \mid L^{\perp}\right) & =\int_{S O_{d}} \mathrm{E} V_{k}\left(\vartheta^{-1} Z_{0} \mid L^{\perp}\right) v(\mathrm{~d} \vartheta) \\
& =\int_{S O_{d}} \mathrm{E} V_{k}\left(Z_{0} \mid \vartheta L^{\perp}\right) v(\mathrm{~d} \vartheta) \\
& =c_{d, k} \mathrm{E} V_{k}\left(Z_{0}\right),
\end{aligned}
$$

where $V_{k}$ on the right-hand side now denotes, more generally, the $k$ th intrinsic volume, and where

$$
c_{d, k}=\kappa_{k} \kappa_{d-k} /\left(\begin{array}{l}
d \\
k
\end{array}\right) \kappa_{d} .
$$

We have obtained the formula

$$
\mathrm{E} f_{d-k}\left(Z_{0}\right)=c_{d, k} \widehat{\gamma_{k}} \mathrm{E} V_{k}\left(Z_{0}\right)
$$

Thus, for the zero cell in the isotropic case, the determination of expected face numbers and of expected intrinsic volumes are equivalent problems. We remark that, for $k=1$, there is a relation connecting the distributions of $f_{d-1}\left(Z_{0}\right)$ and $V_{1}\left(Z_{0}\right)$ (see [7, pp. 177-178]).

In the isotropic case, the value

$$
\mathrm{E} f_{0}\left(Z_{0}\right)=\frac{d !}{2^{d}} \kappa_{d}^{2}
$$

is known (see, e.g. [11, Equation (10.52)]). Since $Z_{0}$ is a.s. a simple polytope, the relation $2 f_{1}=d f_{0}$ gives $\mathrm{E} f_{1}\left(Z_{0}\right)$. The values $\mathrm{E} V_{d}\left(Z_{0}\right)$ and $\mathrm{E} V_{d-1}\left(Z_{0}\right)$ (and second moments) were already determined in [7]. For $d=3$, the Euler relation $f_{0}-f_{1}+f_{2}=2$ further gives $\mathrm{E} f_{2}\left(Z_{0}\right)$ and, thus, $\mathrm{E} V_{1}\left(Z_{0}\right)$. It appears that no other values are known. 


\section{References}

[1] Baumstark, V. and Last, G. (2007). Gamma distributions for stationary Poisson flat processes. Submitted.

[2] Baumstark, V. and Last, G. (2007). Some distributional results for Poisson-Voronoi tessellations. Adv. Appl. Prob. 39, 16-40.

[3] Favis, W. (1995). Extremaleigenschaften und Momente für stationäre Poissonsche Hyperebenenmosaike. Doctoral Thesis, Universität Jena.

[4] Favis, W. (1996). Inequalities for stationary Poisson cuboid processes. Math. Nachr. 178, 117-127.

[5] FAVIS, W. AND WeIss, V. (1998). Mean values of weighted cells of stationary Poisson hyperplane tessellations of $R^{d}$. Math. Nachr. 193, 37-48.

[6] Matheron, G. (1972). Ensembles fermés aléatoires, ensembles semi-markoviens et polyèdres poissoniens. $A d v$. Appl. Prob. 4, 508-541.

[7] Matheron, G. (1975). Random Sets and Integral Geometry. John Wiley, New York.

[8] Miles, R. E. (1961). Random polytopes: the generalisation to $n$ dimensions of the intervals of a Poisson process. Doctoral Thesis, Cambridge University.

[9] Miles, R. E. (1970). A synopsis of 'Poisson flats in Euclidean spaces'. Izv. Akad. Nauk Arm. SSR Ser. Mat. 5, 263-285.

[10] NAGEL, W. (1985). Weighted size distributions for stationary grain models. In Proc. Geobild '85, Wiss. Beiträge der FSU Jena, pp. 118-125.

[11] SCHNEIDER, R. AND WeIL, W. (2008). Stochastic and Integral Geometry. Springer, Berlin.

[12] WeIL, W. (1982). Zonoide und verwandte Klassen konvexer Körper. Monatshefte Math. 94, 73-84.

[13] Weiss, V. (1995). Second-order quantities for random tessellations of $\mathbb{R}^{d}$. Stoch. Stoch. Reports 55, $195-205$. 